\title{
Amplification of MHD waves in swirling astrophysical flows
}

\author{
A. D. Rogava ${ }^{1,2, \star}$, G. Bodo ${ }^{3}$, S. Massaglia ${ }^{1}$, and Z. Osmanov ${ }^{4}$ \\ ${ }^{1}$ Dipartimento di Fisica Generale, Universitá degli Studi di Torino, Via Pietro Giuria 1, Torino 10125, Italy \\ 2 Abdus Salam International Centre for Theoretical Physics, Trieste 34014, Italy \\ 3 Osservatorio Astronomico di Torino, Strada dell'Osservatorio 20, 10025 Pino Torinese, Italy \\ ${ }^{4}$ Centre for Plasma Astrophysics, Abastumani Astrophysical Observatory, Tbilisi-380060, Georgia
}

Received 2 January 2003 / Accepted 18 March 2003

\begin{abstract}
Recently it was found that helical magnetized flows efficiently amplify Alfvén waves (Rogava et al. 2003). This robust and manifold nonmodal effect was found to involve regimes of transient algebraic growth (for purely ejectional flows), and exponential instabilities of both usual and parametric nature. However the study was made in the incompressible limit and an important question remained open - whether this amplification is inherent to swirling MHD flows per se and what is the degree of its dependence on the incompressibility condition. In this paper, in order to clear up this important question, we consider full compressible spectrum of MHD modes: Alfvén waves (AW), slow magnetosonic waves (SMW) and fast magnetosonic waves (FMW). We find that helical flows inseparably blend these waves with each other and make them unstable, creating the efficient energy transfer from the mean flow to the waves. The possible role of these instabilities for the onset of the MHD turbulence, self-heating of the flow and the overall dynamics of astrophysical flows are discussed.
\end{abstract}

Key words. magnetohydrodynamics MHD - waves - instabilities

\section{Introduction}

Lately it became quite commonly presumed and hoped that swirling three-dimensional motion occurs in different kinds of space plasma flows. The presumption is grounded on the mounting evidence from different branches of observational astronomy, while the hope is related to the long-standing aspiration of theoreticians to find in astrophysical flows natural laboratories for testing of their expectations and ideas.

One of the most remarkable observational indications came from Solar and Heliospheric Observatory-Coronal Diagnostic Spectrometer (SOHO-CDS) data that led to the identification (Pike \& Mason 1998) of macrospicules having both rotational and jet-like features. Pike and Mason presented evidence for the existence of blue- and red-shifted emission on either side of an axis of a macrospicule stretching above the limb from a footpoint region on the disk. They interpreted these observations as indicating the presence of a rotation within these tall squalls of hot and magnetized, swirling plasma flows christened as solar tornados - observed both on the limb and the disk of the Sun. This discovery allows to argue (for earlier arguments in the same vein see Pneuman \& Orrall 1986; Shibata \& Uchida 1986) that the wide range of dynamic events in the solar atmosphere, including micro flares, jets, plumes,

Send offprint requests to: A. D. Rogava, e-mail: rogava@ph.unito.it

* On leave from Abastumani Astrophysical Observatory, Kazbegi ave. 2a, Tbilisi-380060, Georgia. surges, spicules and macrospicules, may exhibit complicated, kinematically nontrivial, three-dimensional sheared plasma motions. Generally speaking it looks quite credible that rotation plays a major role in the dynamics of chromospheric and transition region features. It seems plausible to admit that the presence of these complicated patterns of plasma motion deeply influence the dynamics of MHD waves in the solar atmosphere, contribute to the coronal heating, and to the acceleration of the solar wind.

Another message of observational evidence for swirling flows recently came from the totally different class of astronomical objects: Herbig-Haro $(\mathrm{HH})$ jets. Two different observations of young stellar objects in HH 212 (Davis et al. 2000) and DG Tau (Bacciotti et al. 2002), contain serious indications for the presence of rotation in these jets. These results are in accordance with predictions of the popular magnetocentrifugal jet launching model (Blandford \& Payne 1982). The possible impact of these complicated, helical motions on physical processes occurring within these flows is yet to be understood.

The growing evidence in favour of astrophysical helical flows poses a twofold challenge. At the one hand it naturally solicits for the further theoretical study of general aspects - generation, equilibrium, stability and internal dynamics - of helical flows in the framework of plasma astrophysics. At the other hand, after acquiring better understanding of basic physics, it suggests to build concrete prototype models, closely adjusted to specific examples of observed swirling flows, which might 
appear to be useful for shedding some light on the puzzling observational appearance of related astronomical objects.

The basic theory of shear flows tells us that dynamics of waves and vortices, sustained by these flows, are substantially affected by the differential character of motion. On the basic mathematical level these phenomena, somewhat misleadingly christened as "nonmodal processes", are related to the non-self-adjointness of linear dynamics of perturbations in SF (Trefethen et al. 1993). The variety of these processes, that could be labeled as "shear-induced nonmodal processes" (SINP), is quite well-understood for simple, plane parallel flows and thoroughly described in the recent literature (for the latest review see, e.g., Bodo et al. 2001 and references therein). It is known that SINP lead to the generation of new modes of plasma collective behaviour, to new forms of flow-wave, wave-wave and vortex-wave interactions provoked and fueled by "parent" shear flows. These phenomena, originally disclosed in hydrodynamics, take place in various kinds of plasmas and might have a number of astrophysical applications, including pulsar magnetospheric plasmas, solar atmospheric phenomena and galactic gaseous disk dynamics. The interest towards possible applications was strengthened by the recent numerical evidence that real-space appearance of SINP is easily recognizable and robust even in the presence of tangible dissipation (Bodo et al. 2001).

Real astrophysical shear flows are almost always involved in motions with complex kinematics/geometry and kinematic complexity is known to bring an additional variety to SINP. When geometry and kinematics of flows are complex a whole bundle of new effects arises, like "echoing" (repetitive) transient pulsations and different kinds of shear-induced (including parametric) instabilities (Mahajan \& Rogava 1999). The study of kinematically complex SF was initiated in hydrodynamics (Lagnado et al. 1984; Craik \& Criminale 1986; Criminale \& Drazin 1990) and this approach is still largely unknown for the plasma and astrophysics community. Therefore, it is an intriguing challenge and a task of a big practical importance to study these processes in MHD flows of nontrivial geometry and kinematics.

The first step in this direction was recently made (Rogava et al. 2003, hereafter referred as Paper I). In this study the simplifying assumption of incompressibility was adopted, which cuts off modes of acoustic origin (slow magnetosonic waves, SMW, and fast magnetosonic waves FMW) and allows to concentrate on the investigation of the dynamics of Alfvén waves (AW). The subject of the interaction between flows and AW is interesting in a number of astrophysical applications (Balbus \& Hawley 1991; Tagger et al. 1992; Tagger \& Pellat 1999; Ryutova et al. 2001; Varnièr \& Tagger 2002). In Paper I it was found that helical shear flows are efficient amplifiers of AW. In purely ejectional flows (i.e., when no rotation is present) AW are amplified transiently via algebraic, shearinduced instability. In a swirling flow AW are exponentially unstable: depending on the mode of differential motion both usual and parametric instabilities appear.

These results were discussed in the context of their possible (observable) manifestations. It was argued that they might account for the generation of the large-amplitude Alfvén waves - e.g., within "tornado-like" patterns existing in the solar atmosphere. It was suggested that they could lead to the efficient self-heating of flows: the kinetic energy of the flow, being extracted by amplified Alfvén waves, returns back to the flow in the form of the thermal energy because AW are eventually damped via magnetic diffusion. Finally, it was argued that these instabilities might serve as an initial (linear and nonmodal) phase in the ultimate subcritical transition to MHD Alfvénic turbulence in various kinds of astrophysical shear flows.

It is known that the incompressibility condition, used in the Paper I, is quite restrictive. Often the usage of this condition introduces its own imprints on the dynamics of perturbations and it is not trivial to distinguish the genuine SINP from the phenomenological effect imposed by the incompressibility approximation. Therefore it is quite important to consider MHD waves in helical flows without the usage of the incompressibility condition and to study the SINP for the full spectra of MHD waves containing together with the AW also the SMW and the FMW.

This task is undertaken in this paper. We find that the range of processes, sustained by helical flows is extremely rich. It encompasses different kinds of wave transformations, wave beatings. Most importantly, usual and parametric shear instabilities, which has been found in the Paper I, are found to appear for the full spectra of MHD waves containing AW, SMW and FMW. We see that flows of such a high degree of complexity efficiently intertwine all three MHD wave modes and efficiently exchange energy with them. The relevance of these results to the physics of helical flows and to the understanding of possible observational appearance of related astronomical objects are pointed out and critically discussed.

\section{Theory}

Our aim is to study linear collective MHD modes in helical flows. For this purpose we need to write equations of the ideal linearized MHD for the evolution of perturbations within the flow. In Paper I we studied only incompressible perturbations, while now we consider fully compressible case, so our starting equations are $\left[D_{\mathrm{t}} \equiv \partial_{\mathrm{t}}+(\boldsymbol{V} \cdot \nabla)\right]$ :

$D_{\mathrm{t}} \rho+\rho(\nabla \cdot \boldsymbol{V})=0$

$D_{\mathrm{t}} \boldsymbol{V}=-\frac{1}{\rho} \nabla P-\frac{\boldsymbol{B}}{4 \pi \rho} \times(\nabla \times \boldsymbol{B})$,

$D_{\mathrm{t}} \boldsymbol{B}=(\boldsymbol{B} \cdot \nabla) \boldsymbol{V}-\boldsymbol{B}(\nabla \cdot \boldsymbol{V})$,

$\nabla \cdot \boldsymbol{B}=0$.

Our equilibrium model, used in Paper I, assumes a homogeneous MHD plasma ( $\rho_{0}=$ const.), embedded in a homogeneous, vertical magnetic field $\left(\boldsymbol{B}_{0} \equiv\left[0,0, B_{0}=\right.\right.$ const. $\left.]\right)$. We consider instantaneous values of all physical variables as sums of their mean (equilibrium) and perturbational components: $\boldsymbol{B} \equiv \boldsymbol{B}_{0}+\boldsymbol{B}^{\prime}, \rho \equiv \rho_{0}+\rho^{\prime}$, etc. Applying this decomposition we convert (1-4) into the following set for perturbation variables $\left[\mathcal{D}_{\mathrm{t}} \equiv \partial_{\mathrm{t}}+\left(\boldsymbol{U}_{0} \cdot \nabla\right)\right]$ :

$\mathcal{D}_{\mathrm{t}} d+\nabla \cdot \boldsymbol{u}=0$,

$\mathcal{D}_{\mathrm{t}} \boldsymbol{u}+(\boldsymbol{u} \cdot \nabla) \boldsymbol{U}_{0}=-C_{\mathrm{S}}^{2} \nabla d+C_{\mathrm{A}}^{2}\left[\partial_{z} \boldsymbol{b}-\nabla b_{z}\right]$,

$\mathcal{D}_{\mathrm{t}} \boldsymbol{b}=(\boldsymbol{b} \cdot \nabla) \boldsymbol{U}_{0}+\partial_{z} \boldsymbol{u}+\boldsymbol{e}_{z}(\nabla \cdot \boldsymbol{u})$,

$\nabla \cdot \boldsymbol{b}=0$, 
with $d \equiv \rho^{\prime} / \rho_{0}$ and $\boldsymbol{b} \equiv \boldsymbol{B}^{\prime} / B_{0}$. Note also that for compressible perturbations $p^{\prime}=C_{\mathrm{s}}^{2} \rho^{\prime}$ with $C_{\mathrm{s}}$ the homogeneous speed of sound.

If we introduce a new vector quantity $\boldsymbol{h} \equiv \boldsymbol{b}-\boldsymbol{e}_{z} d$ then the above system can effectively be reduced to the following set of second-order equations:

$\mathcal{D}_{\mathrm{t}}^{2} \boldsymbol{h}-\left[(\boldsymbol{h} \cdot \nabla) \boldsymbol{U}_{0} \cdot \nabla\right] \boldsymbol{U}_{0}-\left(C_{\mathrm{s}}^{2}+C_{\mathrm{A}}^{2}\right) \Delta \boldsymbol{h}=$

$C_{\mathrm{A}}^{2}\left[\partial_{z}^{2} \boldsymbol{h}-\nabla\left(\partial_{z} h_{z}\right)-\boldsymbol{e}_{z} \partial_{z}(\nabla \cdot \boldsymbol{h})\right]$,

which describes evolution of all three MHD modes - SMW, AW and FMW - influenced by the presence of the equilibrium flow with an arbitrary $\boldsymbol{U}_{0}$.

In Paper I we worked with the velocity field specified by

$\boldsymbol{U}(r) \equiv[0, r \Omega(r), U(r)]$,

with $\Omega(r)=\mathcal{A} / r^{n}$, where $r=\left(x^{2}+y^{2}\right)^{1 / 2}$ is a distance from the rotation axis, while $\mathcal{A}$ and $n$ are some constants. In particular, $n=0$ and $\mathcal{A}=\Omega_{0}$ for rigidly rotating plasmas, while $n=3 / 2$ and $\mathcal{A}=(G M)^{1 / 2}$, for the Keplerian rotation.

The linear shear matrix for this kind of $\boldsymbol{U}(r)$ has the following form (Rogava et al. 2003):

$\mathcal{S}=\left(\begin{array}{ccc}\sigma & A_{1} & 0 \\ A_{2} & -\sigma & 0 \\ C_{1} & C_{2} & 0\end{array}\right)$.

The key equation of the "nonmodal" method (Craik \& Criminale 1986; Mahajan \& Rogava 1999),

$\partial_{\mathrm{t}} \boldsymbol{k}+\mathcal{S}^{\mathrm{T}} \cdot \boldsymbol{k}=0$

giving a full evolutionary picture of $\boldsymbol{k}$ 's, transcribes in this case to the following set of equations ${ }^{1}$ :

$k_{x}^{(1)}+\sigma k_{x}+A_{2} k_{y}+C_{1} k_{z}=0$,

$k_{y}^{(1)}+A_{1} k_{x}-\sigma k_{y}+C_{2} k_{z}=0$,

while $k_{z}=$ const.

These equations imply that, while $k_{x}(t)$ and $k_{y}(t)$ may have algebraic, exponential or periodic time dependence. For swirling flows the differential rotation parameter $n$ plays decisive role in determining the evolution scenario for the wave number vector $|\boldsymbol{k}(t)|$ : when $n<1$ (including the rigid rotation case) the time evolution of the $|\boldsymbol{k}(t)|$ is periodic, while when $n>1$ (including the Keplerian rotation regime) $|\boldsymbol{k}(t)|$ evolves exponentially.

Note that the following nonlinear combination of $k_{x}(t)$ and $k_{y}(t)$ :

$$
\begin{aligned}
\Delta & \equiv k_{x} k_{y}^{(1)}-k_{y} k_{x}^{(1)}+k_{z}\left(C_{1} k_{y}-C_{2} k_{x}\right) \\
& =A_{2} K_{y}^{2}-A_{1} k_{x}^{2}+2 \sigma k_{x} k_{y}+2 k_{z}\left(C_{1} k_{y}-C_{2} k_{x}\right)=\text { const. }
\end{aligned}
$$

is a conserved quantity. In the case of pure, two-dimensional rotation $\left(k_{z}=C_{1}=C_{2}=0\right)$ it reduces to the constant $\Delta_{\mathrm{r}} \equiv k_{x} k_{y}^{(1)}-k_{y} k_{x}^{(1)}$ (Mahajan \& Rogava 1999), while for the case of a pure outflow $\left(A_{1}=A_{2}=\sigma=0\right)$ it reduces to the conservation of the quantity $\Delta_{\mathrm{e}} \equiv C_{1} k_{y}-C_{2} k_{x}$ (Rogava et al. 2000).

\footnotetext{
${ }^{1}$ Hereafter $F^{(n)}$ will denote $n$th order time derivative of a function $F$.
}

Therefore, we see that the wave-number invariant $\Delta$ in the case of the helical flow is the sum of the $\Delta_{\mathrm{r}}$ and the $\Delta_{\mathrm{e}}$ functions.

Using the same method as in Paper I (see for details also Mahajan \& Rogava 1999) we can effectively convert the system (5)-(8) to the set of first order ordinary differential equations $[\boldsymbol{H} \equiv i \boldsymbol{h}, D \equiv i d]$ :

$D^{(1)}=\boldsymbol{k} \cdot \boldsymbol{u}$,

$\boldsymbol{u}^{(1)}+\mathcal{S} \cdot \boldsymbol{u}=-\left(C_{\mathrm{s}}^{2}+C_{\mathrm{A}}^{2}\right) \boldsymbol{k} D+C_{\mathrm{A}}^{2}\left[k_{z} \boldsymbol{H}-\boldsymbol{k} H_{z}+\boldsymbol{e}_{z} k_{z} D\right]$,

$\boldsymbol{H}^{(1)}=\mathcal{S} \cdot \boldsymbol{H}-k_{z} \boldsymbol{u}$,

$\boldsymbol{k} \cdot \boldsymbol{H}=-k_{z} D$,

Note that (14)-(17) contain two first order ordinary differential equations with time-dependent coefficients. Time-dependence of these coefficients is completely determined by the temporal evolution of the wave number vector $\boldsymbol{k}(t)$ and, therefore, governed by Eq. (12). We can reduce this set to the set of second order equations for the components of the vector $\boldsymbol{H}$, which is the nonmodal form of the Eq. (9). It can be written in the following (vector) form:

$$
\begin{aligned}
\boldsymbol{H}^{(2)} & +C_{\mathrm{s}}^{2} \boldsymbol{k}(\boldsymbol{k} \cdot \boldsymbol{H})-\mathcal{S}^{2} \cdot \boldsymbol{H}+C_{\mathrm{A}}^{2}\left[\left(\boldsymbol{k}-k_{z} \boldsymbol{e}_{z}\right)(\boldsymbol{k} \cdot \boldsymbol{H})\right. \\
& \left.+\left(k_{z}^{2} \boldsymbol{H}-\boldsymbol{k} k_{z} H_{z}\right)\right]=0 .
\end{aligned}
$$

Note that in the helical flow, specified by the shear matrix (11), the square of the matrix is equal to (Rogava et al. 2003):

$\left\|\mathcal{S}^{2}\right\|=\left(\begin{array}{ccc}\Gamma^{2} & 0 & 0 \\ 0 & -\Gamma^{2} & 0 \\ \varepsilon_{1} & \varepsilon_{2} & 0\end{array}\right)$,

where we use notation: $\Gamma \equiv\left(\sigma^{2}+A_{1} A_{2}\right)^{1 / 2}, \varepsilon_{1} \equiv A_{2} C_{2}+\sigma C_{1}$ and $\varepsilon_{2} \equiv A_{1} C_{1}-\sigma C_{2}$. Note that $\left\|\mathcal{S}^{3}\right\|=\Gamma^{2}\|\mathcal{S}\|$.

Splitting both $\boldsymbol{H}$ and $\boldsymbol{k}$ vectors into their longitudinal and transverse components $-\boldsymbol{H} \equiv\left(\boldsymbol{H}_{\perp}, H_{z}\right), \boldsymbol{k} \equiv\left(\boldsymbol{k}_{\perp}, k_{z}\right)-$ we can write more explicit form of (18) revealing the nature of coupling between the MHD modes, imposed by the presence of the velocity shear:

$$
\begin{aligned}
H_{z}^{(2)}+ & C_{\mathrm{s}}^{2} k_{z}^{2} H_{z}+C_{\mathrm{s}}^{2} k_{z}\left(\boldsymbol{k}_{\perp} \cdot \boldsymbol{H}_{\perp}\right)-\left(\varepsilon_{1} H_{x}+\varepsilon_{2} H_{y}\right)=0, \\
\boldsymbol{H}_{\perp}^{(2)}+ & {\left[C_{\mathrm{A}}^{2} k_{z}^{2}-\Gamma^{2}\right] \boldsymbol{H}_{\perp}+\left(C_{\mathrm{s}}^{2}+C_{\mathrm{A}}^{2}\right) \boldsymbol{k}_{\perp}\left(\boldsymbol{k}_{\perp} \cdot \boldsymbol{H}_{\perp}\right) } \\
& +C_{\mathrm{s}}^{2} \boldsymbol{k}_{\perp} k_{z} H_{z}=0 .
\end{aligned}
$$

It is easy to see that in the absence of the shear flow Eqs. (20)-(21) give standard expressions for the dispersion properties of all these modes. The much simpler version of this system was analyzed before for plane-parallel SF of standard MHD plasmas (Chagelishvili et al. 1996). In the astrophysical context the similar kind of coupled ODE's were studied for MHD waves in the solar wind plasmas (Poedts et al. 1998), in galactic gaseous discs (Rogava et al. 1999) and in cylindrical rotationless flux tubes (Rogava et al. 2000). The factual "route" of the wave number vector $\boldsymbol{k}(t)$ temporal "drift" (caused, in its turn, by the differential character of the plasma motion) plays the crucial role in the time evolution of physical perturbations. In parallel flows $\boldsymbol{k}_{\perp}(t)$ exhibits linear time dependence. However, even in this relatively simple case we have the whole set of SINP (Rogava et al. 2000): waves exchange energy with 
the flow and the velocity shear makes waves coupled with one another, making possible their reciprocal transformations.

Helical flows are expected to exhibit evolutionary regimes characteristic to both purely ejectional and purely rotational flow patterns. However, we expect that shear-induced wave transformations (SIT) are more distinctly exhibited by parallel flows without rotation in the transverse plane, because these flows are stable and SIT is the only major kind of SINP occurring in the flow. In helical flows, presumably featuring different sorts of shear-induced instabilities, SIT could be less- or notpronounced. It should be noted that the parameters $\varepsilon_{1}$ and $\varepsilon_{2}$ exist only when both rotation and outflow are present and they are nonzero only when the forces that determine the kinematic portrait of the flow are non-conservative (Craik \& Criminale 1986). Thus their role in the dynamics of MHD waves sustained by swirling flows can be quite significant.

\section{Discussion}

The evolution of linear MHD modes in kinematically complex helical flows might be quite complicated. Before considering any special and/or particular cases we have to recall the following three levels of complexity arising within this problem.

First, the medium itself is complex enough, because it sustains three different linear modes of oscillations: SMW, AW, and FMW. Although in the absence of shear these modes are decoupled, still an arbitrary perturbation excited within this medium is normally a superposition of these three normal modes (Sturrock 1994).

Second, in the presence of a simple, plane-parallel flow with a linear velocity profile: (a) one mode - FMW - becomes able to draw energy out of the background flow; (b) depending on the plasma- $\beta$ different waves become coupled and are able to transform into each other; (c) the system starts exhibiting beat wave phenomena. The resulting picture of the MHD wave dynamics becomes considerably complex (Rogava et al. 2000).

Third, when incompressibility condition is used and the presence of the rotation and stretching of flow lines in the transverse cross section of the flow is "allowed" one finds that new kinds of shear instabilities emerge (Rogava et al. 2003). This is expected to happen also for compressible (acoustic) wave modes, because even in the simplest two-dimensional, nonmagnetized flow with kinematic complexity (Mahajan \& Rogava 1999) usual and parametric instabilities do appear together with asymptotically persistent, "echoing" solutions.

Therefore it seems reasonable to suppose that helical MHD flows, possessing all these levels (or degrees) of complexity plus the complexity of the specifically helical nature, related with the existence of the $\varepsilon_{1}$ and $\varepsilon_{2}$ "helical" parameters, must exhibit highly complicated collective processes, dominated by different regimes of shear-induced variability and instability.

For numerical purposes it is more suitable to deal with the dimensionless version of the (14)-(17) set:

$\varrho^{(1)}=\mathcal{K}_{x}(\tau) v_{x}+\mathcal{K}_{y}(\tau) v_{y}+v_{z}$,

$v_{x}^{(1)}+\Sigma v_{x}+a_{1} v_{y}=-\mathcal{K}_{x}(\tau) \epsilon^{2} \varrho+b_{x}-\mathcal{K}_{x}(\tau) b_{z}$,

$v_{y}^{(1)}+a_{2} v_{x}-\Sigma v_{y}=-\mathcal{K}_{y}(\tau) \epsilon^{2} \varrho+b_{y}-\mathcal{K}_{y}(\tau) b_{z}$,

$$
\begin{aligned}
& v_{z}^{(1)}+R_{1} v_{x}+R_{2} v_{y}=-\epsilon^{2} \varrho, \\
& b_{x}^{(1)}=\Sigma b_{x}+a_{1} b_{y}-v_{x}, \\
& b_{y}^{(1)}=a_{2} b_{x}-\Sigma b_{y}-v_{y}, \\
& \mathcal{K}_{x}(\tau) b_{x}+\mathcal{K}_{y}(\tau) b_{y}+b_{z}=0,
\end{aligned}
$$

derived from (14)-(17) with the usage of dimensionless notation: $\tau \equiv C_{\mathrm{A}} k_{z} t, \mathcal{K}_{x}(\tau) \equiv k_{x}(t) / k_{z}, \mathcal{K}_{y}(\tau) \equiv k_{y}(t) / k_{z}, v_{i} \equiv u_{i} / C_{\mathrm{A}}$, $\Sigma \equiv \sigma / C_{\mathrm{A}} k_{z}, a_{1,2} \equiv A_{1,2} / C_{\mathrm{A}} k_{z}, R_{1,2} \equiv\left(C_{1,2} / k_{z} C_{\mathrm{A}}\right), \epsilon \equiv C_{\mathrm{s}} / C_{\mathrm{A}}$.

It is instructive to calculate how the total energy of perturbations being, in this case, the sum of kinetic, compressional and magnetic energies:

$E_{\text {tot }} \equiv E_{\text {kin }}+E_{\mathrm{c}}+E_{\mathrm{m}}$,

$E_{\mathrm{kin}} \equiv\left(v_{x}^{2}+v_{y}^{2}+v_{z}^{2}\right) / 2$,

$E_{\mathrm{c}} \equiv \epsilon^{2} \varrho^{2} / 2$,

$E_{\mathrm{m}} \equiv\left(b_{x}^{2}+b_{y}^{2}+b_{z}^{2}\right) / 2$,

changes in time. We can easily see that the evolutionary equation for the total energy is:

$$
\begin{aligned}
E^{(1)}= & \left(a_{1}+a_{2}\right)\left(b_{x} b_{y}-v_{x} v_{y}\right)+\Sigma\left[\left(v_{y}^{2}-v_{x}^{2}\right)+\left(b_{x}^{2}-b_{y}^{2}\right)\right] \\
& +R_{1}\left(b_{x} b_{z}-v_{x} v_{z}\right)+R_{2}\left(b_{y} b_{z}-v_{y} v_{z}\right) .
\end{aligned}
$$

The scope of this paper is not full investigation of all possible regimes of evolution. Instead, our purpose is to see whether shear instabilities, disclosed in the incompressibility limit for AW, appear also in the compressible case, for the blend of SMW, AW, and FMW. It is instructive and convenient to unfold these phenomena by following the scheme used in our previous studies of cylindrical flows (Rogava et al. 2000 and the Paper I) and to see how two, major regimes of $\boldsymbol{k}(t)$-dynamics (periodic and exponential) affect the course of collective phenomena in flows with different values of the plasma- $\beta$.

\subsection{High- $\beta$ plasmas}

In parallel flows, when $\epsilon^{2} \gg 1$, FMW are decoupled from the AW and SMW, while the latter two are coupled (Rogava et al. 2000) and may transform into each other. Swirling flows show more complicated behaviour. When $\gamma^{2} \equiv$ $\left(\Gamma / C_{A} k_{z}\right)^{2}<0$, i.e., when temporal evolution of wavenumber vectors $\boldsymbol{k}(t)$ is periodic, we encounter with two new effects:

1. The appearance of "echoing" waves, consisting of repetitive, modulated bumps of AW and SMW, exchanging energy with the mean flow. Figure 1 displays an example of such process. From the figure we can surmise that mutual $\mathrm{AW} \rightleftharpoons \mathrm{SMW}$ transformations still happen and the resulting mixture of waves "pulsates" taking and giving energy from/to the background helical flow! This process has well-pronounced quasiperiodic nature.

2. Since coefficients in (22)-(25) vary periodically it is plausible to expect that for certain cases the system must also exhibit some kind of self-parametric instability. The term "self-parametric" seems appropriate, because it is the consequence of the velocity shear inherent to the system and forcing on itself (Argentina et al. 1999). This kind of instability was first discovered for plain acoustic waves in 

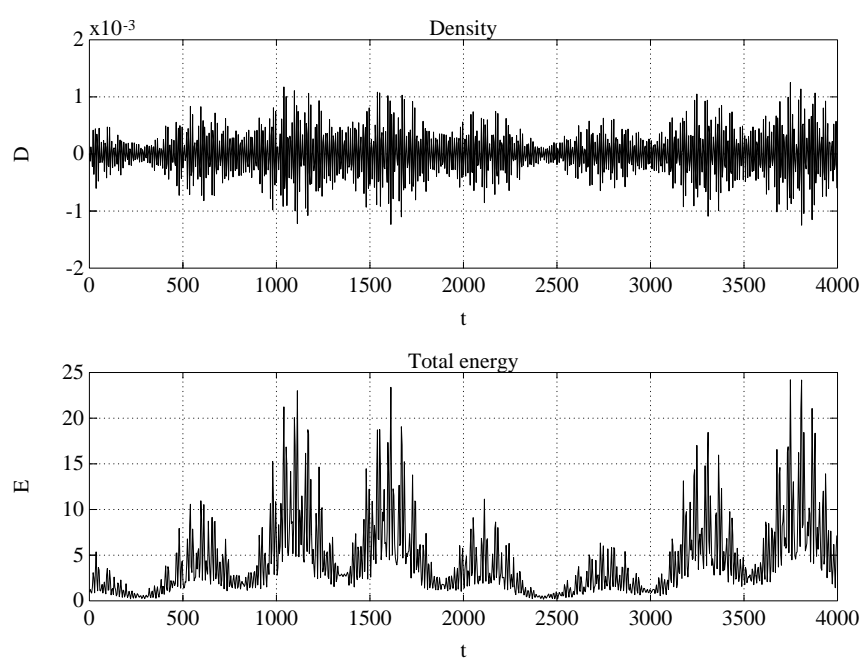

Fig. 1. The temporal evolution of the density $D(\tau)$ and the normalized total energy of perturbations $E_{\mathrm{tot}}(\tau) / E_{\mathrm{tot}}(0)$, which exhibits quasiperiodic and pulsational behavior. The set of parameters is: $\epsilon=10$, $\mathcal{K}_{x}(0)=10, \mathcal{K}_{y}(0)=8, R_{1}=0.8, R_{2}=2, \Sigma=0, a_{1}=-0.1, a_{2}=0.1$.
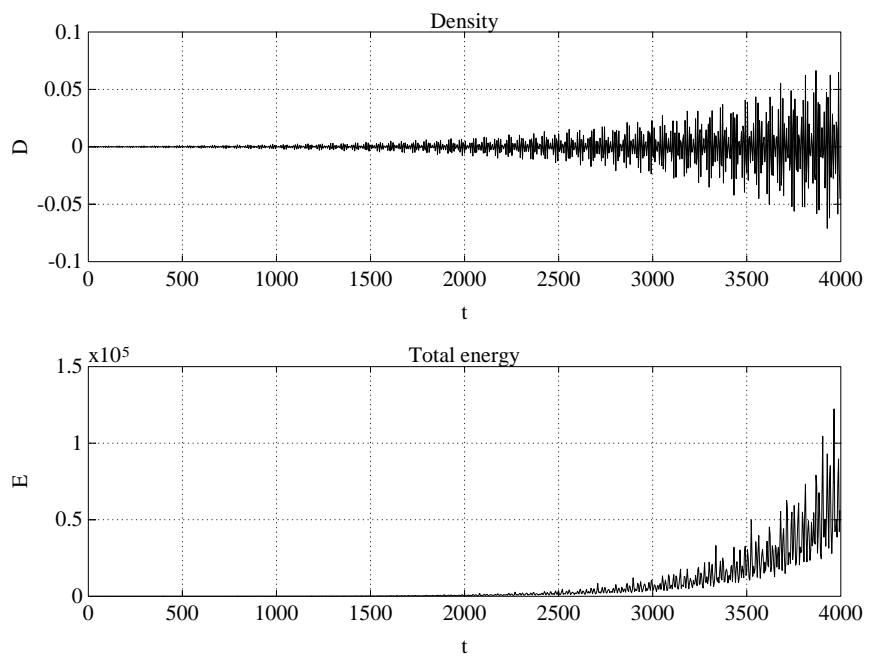

Fig. 2. The evolution of parametrically unstable blend of AW and SMW. The set of parameters is the same as in Fig. 1, except $R_{1}=0.4$.

2-D flow patterns of neutral fluids (Mahajan \& Rogava 1999). Similar sort of instability was found for Alfvén waves in the Paper I. Numerical examination of the (22) (25) allowed us to find similar instabilities for the compressible case. One example is given in the Fig. 2. The values of all parameters (except $R_{1}$ ) here are the same as in the Fig. 1 , but $R_{1}=0.4$. The figure shows that in this case hydromagnetic oscillations amplify exponentially, extracting energy from the flow. Note that for the existence of this kind of parametric instability it is not necessary to have any periodicity in the background flow, but it is essential to have periodic time variation of the wave number vector.

Let us turn our attention, now, to flows with "sharper" rate of differential rotation, with $n>1$. In this case $\gamma^{2}>0$ and the temporal evolution of $\boldsymbol{k}(t)$ 's becomes exponential. In the absence of dissipation these flows host rather robust shear instabilities. The similar kind of instability was found for acoustic waves in
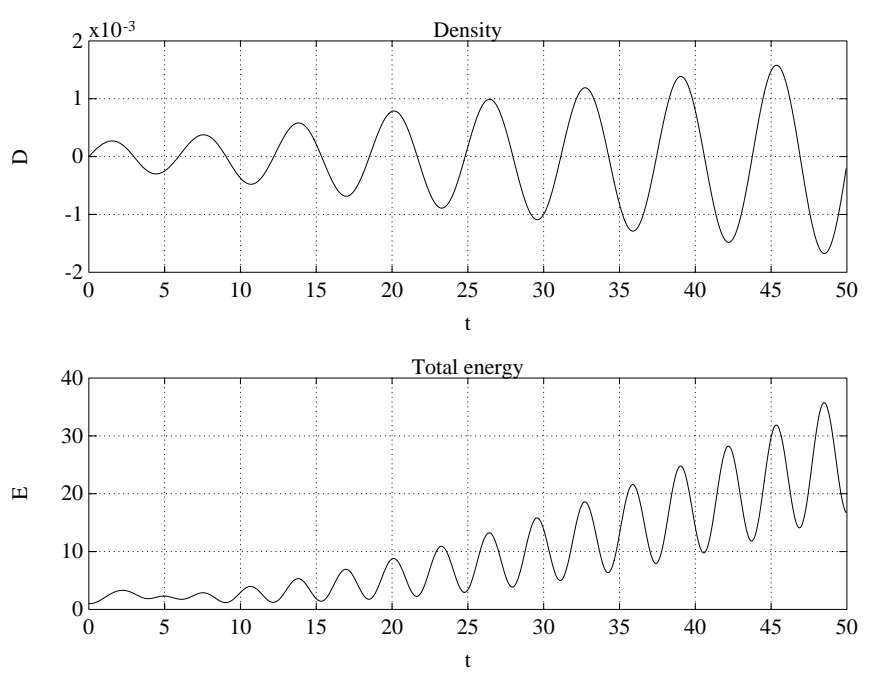

Fig. 3. Shear instability of low-frequency MHD oscillations. The plot features temporal evolution of the same kind of initial value problem as in Fig. 1. The only difference is that The evolution of parametrically unstable blend of AW and SMW. The set of parameters is the same as in Fig. 1, except that $a_{1}=0.1$ now, making the sign of $\gamma^{2}$ positive.

a 2-D flow (Mahajan \& Rogava 1999) and in the Paper I it was detected for Alfvén waves as well. The example of such instability for the mixture of AW and SMW is shown in the Fig. 3. The parameters are the same as for the Fig. 1 , only $a_{1}=0.1$ (it reverses the sign of the $\Lambda^{2}$ ). We see that in this case the mixture of AW and SMW undergoes rather strong, exponential enhancement of its amplitude and energy.

\subsection{Low- $\beta$ plasmas}

In this case hydromagnetic oscillations offer quite a different picture. Namely, the SMW mode is decoupled from the other two MHD modes-its dispersion curve runs well below the Alfvén dispersion "horizontal" and it is not coupled with other two (AW and FMW) modes. The FMW, on the other hand is coupled with the AW. When rotation is absent and there is only parallel outflow the coupling ensures linear transformation of the AW into the FMW (see as an example Figs. 6a-d in Rogava et al. 2000).

In the helical flow, getting certain "input" of initial AW and/or FMW oscillations, these waves will keep transforming into each other. Besides the mixture of waves might exhibit the same kind of "echoing" and unstable behavior as it was seen in high- $\beta$ plasmas. Numerical simulations support this expectation. Making values of $a_{1}$ and $a_{2}$ nonzero $\left(a_{1}=-0.1\right.$ and $a_{2}=0.1$, so that $\gamma^{2}<0$ ) and using following parameters: $R_{1}=0.01$ and $R_{2}=0.9$ we see (Fig. 4) the appearance of interesting patterns of "echoing" solutions with quasiperiodic variability of the perturbation total energy ${ }^{2}$.

We find that parametric instabilities are also characteristic to low- $\beta$ plasmas. The example is given in Fig. 5 . The parametric nature of the instability is apparent from the remarkable narrowness of the range of parameter values, where the

\footnotetext{
${ }^{2}$ Note that here, as elsewhere on the plots of this paper, the total energy is normalized on its initial value.
} 

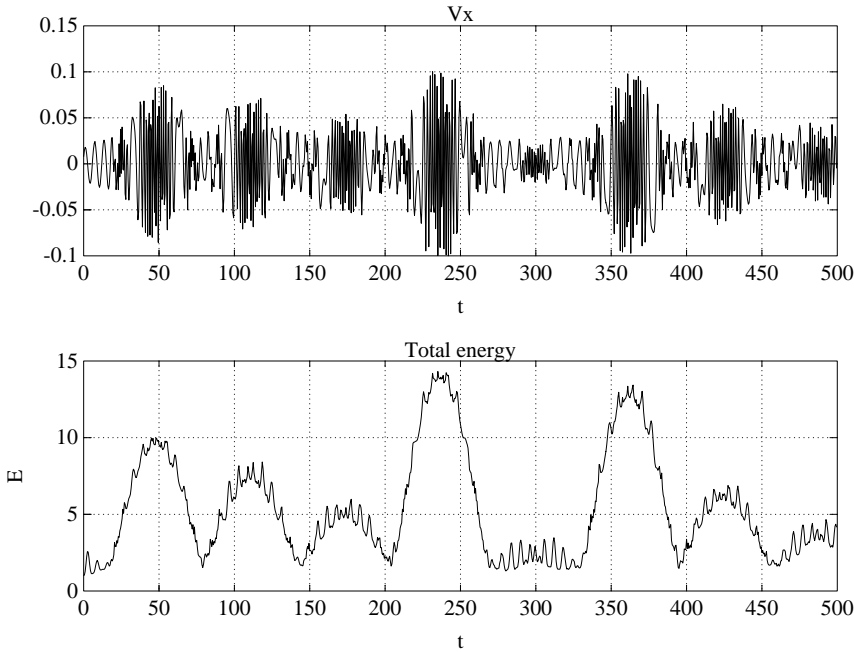

Fig. 4. The temporal evolution of initially excited AW, which became partially transformed into FMW and exhibits quasiperiodic, pulsational behavior in low $\beta$ plasma flow. The set of parameters is: $\epsilon=0.1$, $a_{1}=-0.1, a_{2}=0.1, \Sigma=0, R_{1}=0.01$ and $R_{2}=0.9$. Figure 4 shows time evolution of the velocity perturbation $v_{x}$ and the total energy normalized on its initial value $E_{\text {tot }}(\tau) / E_{\text {tot }}(0)$.
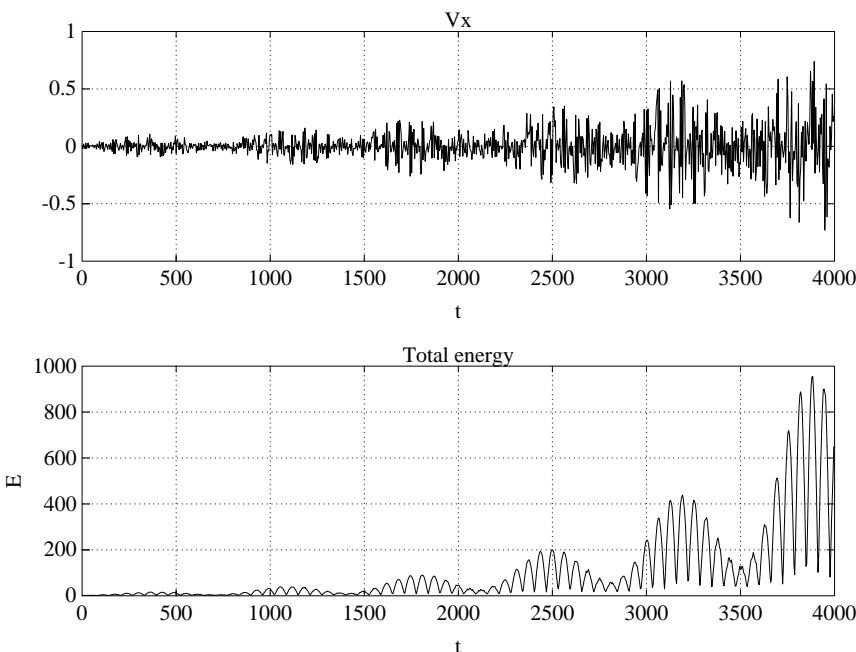

Fig. 5. The parametrically unstable solution. The values of the parameters are: $\epsilon=0.1, a_{1}=-0.1 a_{2}=0.1, \Sigma=0, R_{1}=0.01$ and $R_{2}=0.83$.

instability is present. Note that the set of parameters used for Fig. 5 is the same as for Fig. 4 except $R_{2}=0.83$. If one takes the value of $R_{2}$ less by 0.01 , then the instability disappears and the system again displays the "echoing" behaviour.

When the differential rotation is characterized by $n>1$ profile $\left(\gamma^{2}>0\right)$ the system shows strong exponential shear instability. The corresponding plots are given in Fig. 6.

\subsection{The case of $\beta \simeq 1$}

From the studies of parallel flows we know that in terms of wave couplings and mutual transformations this is the most complex case: all MHD wave modes are coupled and may transform into each other. In helical flows the presence of wave transformations may be less visible, overshadowed (when $\left.\gamma^{2}<0\right)$ by quasiperiodic modulation of waves appearing
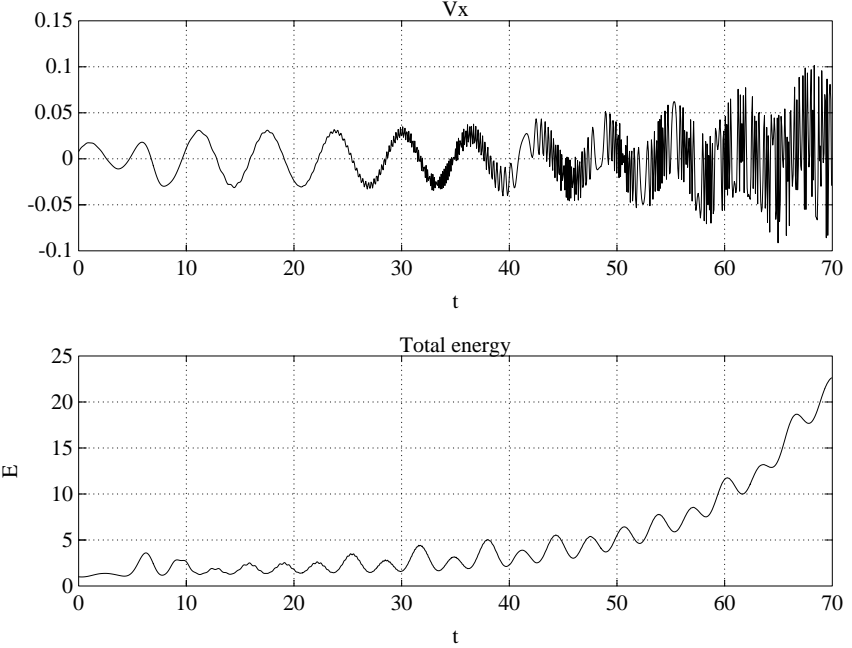

Fig. 6. The shear instability in the $\gamma^{2}>0$ case. The values of the parameters are: $\epsilon=0.1, a_{1}=0.1 a_{2}=0.1, \Sigma=0, R_{1}=1$ and $R_{2}=1$.
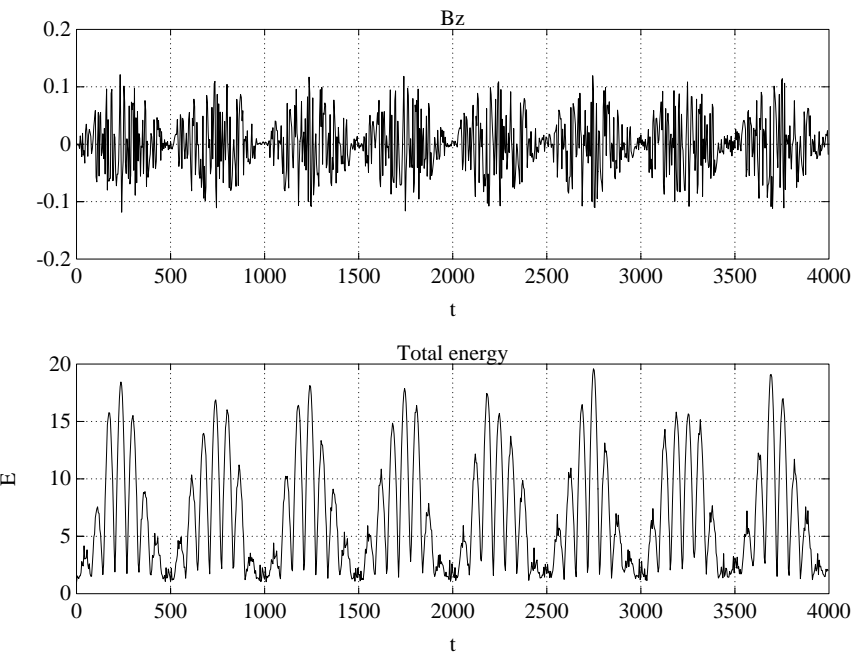

Fig. 7. The temporal evolution of the wave blend that exhibits quasiperiodic, pulsating behavior in $\epsilon=1$ plasma flows. The set of parameters is: $a_{1}=-0.1 a_{2}=0.1, \Sigma=0, R_{1}=0.1$ and $R_{2}=0.85$. The plots show time evolution of the magnetic field $B_{x}$ perturbations and the normalized energy $E_{\mathrm{tot}}(\tau) / E_{\mathrm{tot}}(0)$.

as repetitive, ("echoing") bundles of mixed AW, SMW and FMW modes (see Fig. 7); or by self-parametric instability (see Fig. 8). While when $\gamma^{2}>0$ the waves are unstable in the similar way (see Fig. 9) as in previously considered high- $\beta$ and low- $\beta$ cases.

\section{Conclusion}

The goal of this work was to find out whether the exotic SINP originally found for 2-D kinematically complex velocity patterns of neutral fluids (Mahajan \& Rogava 1999) and for 3-D helical flows of magnetized conducting fluids in the incompressible limit, appear also for the full spectra of MHD waves sustained by compressible MHD medium. We found that this is indeed the case! Therefore we can now firmly claim that the range of the SINP, typical for the flows of complicated, helical nature, is broad. These SINP persist to show up both in the 

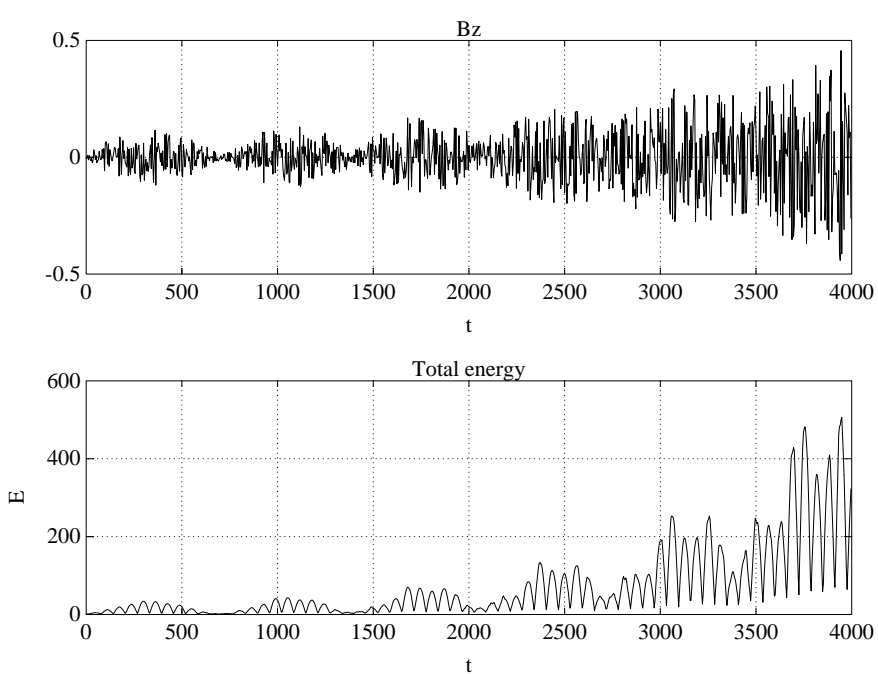

Fig. 8. The evolution of parametrically unstable blend of waves. The set of parameters is: $a_{1}=-0.1 a_{2}=0.1, \Sigma=0, R_{1}=0.1$ and $R_{2}=0.8005$. Note that the range of $R_{2}$ when the evolution of the wave mixture is parametrically unstable is very narrow: [0.8001; 0.8011].

incompressible and compressible cases. They are present in flows with arbitrary values of plasma- $\beta$.

In relatively mildly sheared flows (with $n<1$, i.e., including rigidly rotating systems) MHD modes appear to be rather stable exhibiting either "echoing" pulsational behaviour or relatively long-time-scaled parametric instabilities. One can expect that in helical flows of this nature, especially in wellbeamed, or well-collimated flows such as jets, shear flow effects are not likely to lead to disruptive instabilities. Instead, through quasiperiodic interchange of energy with the mean flow, they might tend to exhibit certain modes of quasi stable and quasiperiodic structuring both in the space and in time.

In more strongly sheared systems $(n>1$ including Keplerian rotation) waves within swirling flows become subject to potentially very fast-growing shear instabilities, which would either lead to the disruption of "parent" flow patterns or to the development of the MHD turbulence with subsequent phase transition to turbulent rotational flow systems. We have to bear in mind that in this case the exponential growth of the $|\boldsymbol{k}(t)|$ inevitably makes the spatial length-scales of perturbations smaller and smaller. It implies that the effects of the viscous decay and/or magnetic diffusion, neglected while we consider the MHD flow as an "ideal" one, must sooner or later become important and lead to the dissipation of the energy gained by the exponentially increasing waves into the heat. It can be argued that in accretion-ejection systems, where the rotational law seems to be quasikeplerian, these instabilities may account for the transition to turbulence in accreted plasma flows. Alternatively, this process might lead to effective "self-heating" of these flows, when the energy acquired by waves from the flows through the agency of the shear instability would eventually transform into heat via diffusion.

Speaking about swirling astrophysical flows we are keen to use the term "cosmic tornado" for this class of flows, because they are reminiscent of powerful and dangerous tornados in the Earth's atmosphere. Recently such
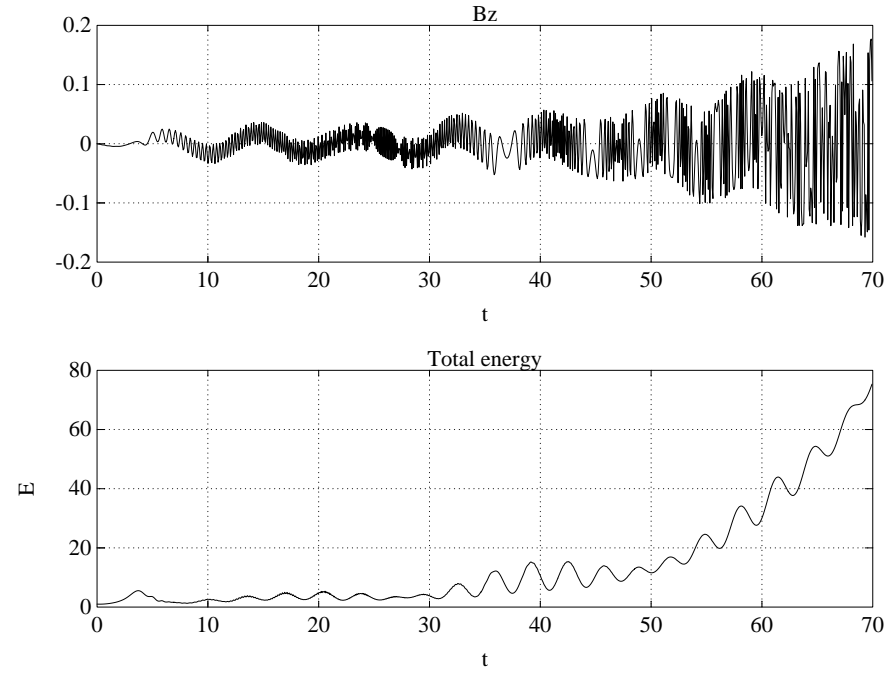

Fig. 9. The shear instability in the $\lambda^{2}>0$ case. The set of parameters is: $a_{1}=0.1 a_{2}=0.1, \Sigma=0, R_{1}=1$ and $R_{2}=1$.

structures, called solar tornados, were identified in the polar regions of the solar atmosphere (both on the limb and the disk) by SOHO-CDS observations (Pike \& Mason 1998). Another class of cosmic tornados are, probably, stellar jets, because recent observational results (Bacciotti et al. 2002; Davis et al. 2000) seem to confirm the predictions of various magnetocentrifugal jet acceleration models (Shu et al. 1995; Ferreira \& Pelletier 1995; Camenzind 1997; Lery et al. 1999; Königl \& Pudritz 2000) about the presence of a swirling motion within the jets. The third class of astrophysical flows with possible presence of tornado-like motion are accretion columns - magnetically channeled shear flows of plasma to a neutron star's (or a white dwarf's) magnetic pole. The infalling matter is decelerated approaching the star surface, but it can also form a shock high above the star's surface (Hujeirat \& Papaloizou 1998). Originally the formation of rotational accretion columns was considered in the astrophysical fluid dynamics context (Cassen 1978), while now accretion columns associated with X-ray pulsars and cataclysmic binaries are modeled either as thin slabs or tall columns of infalling matter. It seems reasonable to surmise that accretion columns comprise 3-D swirling plasma flows.

Bearing in mind these perspectives we should stress that the results of this paper are quite general and (deliberately) not adjusted to either of three kinds of swirling astrophysical flows. For all these classes of "cosmic tornados" the level of our factual knowledge about their basic kinematic features is still inadequate for building of any credible concrete models. However we hope that these future models, based on the gained data and implying real-space sophisticated simulations, will show how the generic processes disclosed in this paper and the Paper I might influence the overall dynamics of real "cosmic tornados".

Acknowledgements. The authors are grateful to Swadesh Mahajan and Stefaan Poedts for valuable discussions. Andria Rogava and Zaza Osmanov are grateful to the International Centre for Theoretical 
Physics for supporting them, in part, through the Regular Associate Membership Award and the Young Collaborator grant, respectively. Andria Rogava is grateful to the Universitá degli Studi di Torino for supporting him, in part, through the Assegno di Ricerca $e$ Collaborazione.

\section{References}

Argentina, M., Coullet, P., \& Risler, E. 1999, Phys. Rev. Lett., 86, 807 Bacciotti, F., Ray, T. P., Mundt, R., Eisloffel, J., \& Solf, J. 2002, ApJ, 576,222

Balbus, S. A., \& Hawley, J. F. 1991, ApJ, 376, 214

Blandford, R. D., \& Payne, D. G. 1982, MNRAS, 199, 883

Bodo, G., Poedts, S., Rogava, A. D., \& Rossi, P. 2001, A\&A, 374, 337

Camenzind, M. 1997, in Harbig-Haro Flows and the Birth of Low Mass Stars, ed. B. Reipurth, \& C. Bertout (Dordrecht: Kluwer), IAU Symp., 182, 241

Cassen, P. 1978, ApJ, 219, 336

Chagelishvili, G. D., Rogava, A. D., \& Tsiklauri, D. G. 1996, Phys. Rev. E, 53, 6028

Craik, A. D. D., \& Criminale, W. O. 1986, Proc. R. Soc. Lond. A, 406, 13

Criminale, W. O., \& Drazin, P. G. 1990, Stud. Appl. Math., 83, 123

Davis, C. J., Berndsen, A., Smith, M. D., Chrysostomou, A., \& Hobson, J. 2000, MNRAS, 314, 241

Ferreira, J., \& Pelletier, G. 1995, A\&A, 295, 807

Hujeirat, A., \& Papaloizou, J. C. B. 1998, A\&A, 340, 593
Königl, A., \& Pudritz, R. E. 2000, in Protostars and Planets IV, ed. V. M. Annings, A. P. Boss, \& S. S. Russell (Tucson: Univ. Arizona Press), 759

Lagnado, R. R., Phan-Thien, N., \& Leal, L. G. 1984, Phys. Fluids, 27, 1094

Lery, T., Henriksen, R. N., \& Fiege, J. D. 1999, A\&A, 350, 254

Mahajan, S. M., \& Rogava, A. D. 1999, ApJ, 518, 814

Pike, C. D., \& Mason, H. E. 1998, Sol. Phys., 182, 333

Pneuman, G. W., \& Orrall, F. Q. 1986, in Physics of the Sun, vol. II, ed. P. A. Sturrock (Dordrecht: Reidel), 71

Poedts, S., Rogava, A. D., \& Mahajan, S. M. 1998, ApJ, 505, 369

Rogava, A. D., Poedts, S., \& Heirman, S. 1999, MNRAS, 307, L31

Rogava, A. D., Poedts, S., \& Mahajan, S. M. 2000, A\&A, 354, 749

Rogava, A. D., Mahajan, S. M., Bodo, G., \& Massaglia, S. 2003, A\&A, 399, 421

Ryutova, M., Habbal, S., Woo, R., \& Tarbell, T. 2001, Sol. Phys., 200, 213

Shibata, K., \& Uchida, Y. 1986, Sol. Phys., 103, 299

Shu, F. H., Najita, J. R., Ostriker, E., \& Shang, H. 1995, ApJ, 455, L155

Sturrock, P. A. 1994, Plasma Physics (Cambridge: Cambridge University Press)

Tagger, M., Pellat, R., \& Coroniti, F. V. 1992, ApJ, 393, 708

Tagger, M., \& Pellat, R. 1999, A\&A, 349, 1003

Trefethen, L. N., Trefethen, A. E., Reddy, S. C., \& Driscoll, T. A. 1993, Science, 261, 578

Varnière, P., \& Tagger, M. 2002, A\&A, 394, 329 